\title{
Vacuum polarization of a quantized scalar field in the thermal state in a long throat
}

\author{
Arkady A. Popov \\ Kazan Federal University, 18 Kremlyovskaya St., Kazan 420008, Russia
}

\begin{abstract}
Vacuum polarization of scalar fields in the background of a long throat is investigated. The field is assumed to be both massive or massless, with arbitrary coupling to the scalar curvature, and in a thermal state at an arbitrary temperature. Analytical approximation for $\left\langle\varphi^{2}\right\rangle_{r e n}$ is obtained.

PACS numbers: 04.62.+v, 04.70.Dy
\end{abstract}




\section{INTRODUCTION}

The study of vacuum polarization effects in strong gravitational fields is a pertinent issue since such effects may play a role in the cosmological scenario and in the construction of a self-consistent model of black hole evaporation. These effects may be taken into account by solving the semiclassical backreaction equations,

$$
G_{\nu}^{\mu}=8 \pi\left\langle T_{\nu}^{\mu}\right\rangle_{r e n}
$$

where $\left\langle T_{\nu}^{\mu}\right\rangle_{\text {ren }}$ is the expectation value of the stress-energy tensor operator for the quantized fields. Possible vacuum fluctuations of quantized fields can create wormholes [1 4 4].

The main difficulty in the theory of semiclassical gravity is that the effects of the quantized gravitational field are ignored. The popular solution to this problem is to justify ignoring the gravitational contribution by working in the limit of a large number of fields, in which the gravitational contribution is negligible. Another problem is that the vacuum polarization effects are determined by the topological and geometrical properties of spacetime as a whole or by the choice of quantum state in which the expectation values are taken. It means that calculation of the functional dependence of $\left\langle T_{\nu}^{\mu}\right\rangle_{\text {ren }}$ on the metric tensor in an arbitrary spacetime presents formidable difficulty. Only in some spacetimes with high degrees of symmetry for the conformally invariant fields $\left\langle T_{\mu \nu}\right\rangle_{\text {ren }}$ can be computed and equations (1) can be solved exactly $\left[\begin{array}{ll}5 & -9\end{array}\right]$.

Numerical computations of $\left\langle T_{\nu}^{\mu}\right\rangle_{\text {ren }}$ are usually extremely intensive [10 17]. In some cases $\left\langle T_{\mu \nu}\right\rangle_{r e n}$ is determined by the local properties of a spacetime and it is possible to calculate the functional dependence of the renormalized expression for the vacuum expectation value of the stress-energy tensor operator of the quantized fields on the metric tensor approximately (too long). One of the most widely known examples of such a situation is the case of a very massive field. In this case $\left\langle T_{\nu}^{\mu}\right\rangle_{\text {ren }}$ can be expanded in terms of powers of the small parameter

$$
\frac{1}{m l} \ll 1
$$

where $m$ is the mass of the quantized field and $l$ is the characteristic scale of the spacetime curvature [18-24].

Approximate calculations for conformally coupled massless fields have also been made. For $\left\langle T_{\nu}^{\mu}\right\rangle$ in static Einstein spacetimes $\left(R_{\mu \nu}=\Lambda g_{\mu \nu}\right)$ these include the approximations of 
Page, Brown, and Ottewill [25 27]. These results have been generalized to arbitrary static spacetimes by Zannias [28]. A different approach to the derivation of approximate expressions for $\left\langle\varphi^{2}\right\rangle$ and $\left\langle T_{\nu}^{\mu}\right\rangle$ for conformally coupled massless fields in static spacetimes has been proposed by Frolov and Zel'nikov [29]. Their calculations were based primarily on geometric arguments and the common properties of the stress-energy tensor rather than on a field theory. Using the methods of quantum field theory the expressions for $\left\langle\varphi^{2}\right\rangle$ and $\left\langle T_{\nu}^{\mu}\right\rangle$ of a scalar field in static spherically symmetric asymptotically flat spacetimes have been obtained by Anderson, Hiscock, and Samuel [10]. They assumed that the field is massive or massless with an arbitrary coupling $\xi$ to the scalar curvature and in a zero temperature quantum state or a nonzero temperature thermal state. The result was presented as a sum of two parts, numerical and analytical:

$$
\left\langle T_{\nu}^{\mu}\right\rangle_{\text {ren }}=\left\langle T_{\nu}^{\mu}\right\rangle_{\text {numeric }}+\left\langle T_{\nu}^{\mu}\right\rangle_{\text {analytic }}
$$

The analytical part of their expression is conserved. This has a trace equal to the trace anomaly for the conformally invariant field. For these reasons they proposed to use $\left\langle T_{\nu}^{\mu}\right\rangle_{\text {analytic }}$ directly as an approximation for $\left\langle T_{\nu}^{\mu}\right\rangle_{\text {ren }}$. An analogous result has been obtained by Groves, Anderson, and Carlson [30] in the case of a massless spin $\frac{1}{2}$ field in a general static spherically symmetric spacetime.

Let us stress that the single parameter of length dimensionality in problem (1) is the Planck length $l_{P 1}$. This implies that the characteristic scale $l$ of the spacetime curvature (which corresponds to the solution of equations (1D) can differ from $l_{P l}$ only if there is a large dimensionless parameter. As an example of such a parameter one can consider a number of fields the polarization of which is a source of spacetime curvature ${ }^{1}$. In the case of massive field, the existence of an additional parameter $1 / m$ does not increase the characteristic scale of the spacetime curvature $l$ which is described by the solution of equations (11) ${ }^{2}$. For the massless quantized fields such a parameter can be the coupling constants of field to the curvature of spacetime [4]. Another possibility of introducing an additional parameter in the problem (11) is to consider the non-zero temperature of quantum state for the quantized

\footnotetext{
${ }^{1}$ Here and below it is assumed, of course, that the characteristic scale of change of the background gravitational field is sufficiently greater than $l_{P 1}$ so that the very notion of a classical spacetime still has some meaning.

${ }^{2}$ The characteristic scale of the components $G_{\nu}^{\mu}$ on the left-hand side of equations (1) is $1 / l^{2}$, on the right-hand side $-l_{P l}^{2} /\left(m^{2} l^{6}\right)$
} 
field. It is known (see, e.g., [31]) that in the high-temperature limit (when $T \gg 1 / l, T$ being a temperature of thermal state) $\left\langle T_{\nu}^{\mu}\right\rangle$ for such a thermal state is proportional to the fourth power of the temperature $T$.

In this paper, approximate expression for $\left\langle\varphi^{2}\right\rangle_{\text {ren }}$ of a quantized scalar field in the background of a long throat is derived. The field is assumed to be both massless or massive with a coupling $\xi$ to the scalar curvature and in a thermal state at an arbitrary temperature.

In Sec. II the expression for the Euclidian Green's function of a scalar field in a general static spherically symmetric spacetime is derived. In Sec. III the WKB approximation for $\left\langle\varphi^{2}\right\rangle_{\text {unren }}$ is obtained and the renormalization procedure is described. The results are summarized in Sec. IV. Here we give also an example of the evaluation $\left\langle\varphi^{2}\right\rangle_{\text {ren }}$ near ultraextremal horizon. In the Appendixes some technical results are derived and long expressions are displayed.

The units $\hbar=c=G=k_{B}=1$ are used throughout the paper.

\section{AN UNRENORMALIZED EXPRESSION FOR $\left\langle\varphi^{2}\right\rangle$}

The metric of the static spherically symmetric spacetime is analytically continued into Euclidean form

$$
d s^{2}=f d \tau^{2}+d \rho^{2}+r^{2}\left(d \theta^{2}+\sin ^{2} \theta d \varphi^{2}\right)
$$

where $f$ and $r$ are functions of $\rho$, and $\tau$ is the Euclidean time $(\tau=i t$, where $t$ is the coordinate corresponding to the timelike Killing vector, which always exists in static spacetime).

In this paper, the point-splitting method is employed for the regularization of ultraviolet divergences. The expectation value $\left\langle\phi^{2}(x)\right\rangle$ is found from the coincidence limit of the Euclidean Greens function $G_{E}(x, x) \equiv \lim _{\tilde{x} \rightarrow x} G_{E}(x, \tilde{x})$. For a scalar field in a spacetime given by Eq. (41), $G_{E}(x, \tilde{x})$ satisfies

$$
\left[\square_{x}-m^{2}-\xi R(x)\right] G_{E}(x, \tilde{x})=-\frac{\delta^{4}(x, \tilde{x})}{\sqrt{g(x)}},
$$

where $\square_{x}$ is the Laplace-Beltrami operator of the Euclidean metric corresponding to Eq. (4), $m$ is the mass of scalar field $\phi$ with coupling $\xi$ to the scalar curvature $R$ of spacetime.

The representation for the Euclidean Green's function $G_{E}(x, \tilde{x})$ of a scalar field in a static 
spherically symmetric spacetime used by Anderson et al. [10] is the following:

$$
\begin{aligned}
G_{E}(x ; \tilde{x})= & \frac{T}{4 \pi} \sum_{l=0}^{\infty}(2 l+1) P_{l}(\cos \gamma) C_{0 l} p_{0 l}\left(\rho_{<}\right) q_{0 l}\left(\rho_{>}\right) \\
& +\frac{T}{2 \pi} \sum_{n=1}^{\infty} \cos [2 \pi n T(\tau-\tilde{\tau})] \sum_{l=0}^{\infty}(2 l+1) P_{l}(\cos \gamma) C_{n l} p_{n l}\left(\rho_{<}\right) q_{n l}\left(\rho_{>}\right),
\end{aligned}
$$

where $P_{l}$ is a Legendre polynomial, $T$ is the temperature of the field, $\cos \gamma \equiv \cos \theta \cos \tilde{\theta}+$ $\sin \theta \sin \tilde{\theta} \cos (\varphi-\tilde{\varphi}), C_{n l}$ is a normalization constant, $\rho_{<}$and $\rho_{>}$represent the lesser and greater of $\rho$ and $\tilde{\rho}$, respectively, and the modes $p_{n l}(\rho)$ and $q_{n l}(\rho)$ satisfy the equation

$$
\left\{\frac{d^{2}}{d \rho^{2}}+\left[\frac{1}{2 f} \frac{d f}{d \rho}+\frac{1}{r^{2}} \frac{d r^{2}}{d \rho}\right] \frac{d}{d \rho}-\left[\frac{(2 \pi n T)^{2}}{f}+\frac{l(l+1)}{r^{2}}+m^{2}+\xi R\right]\right\}\left\{\begin{array}{l}
p_{n l} \\
q_{n l}
\end{array}\right\}=0 .
$$

Normalization of $p_{n l}$ and $q_{n l}$ is given by the Wronskian condition

$$
C_{n l}\left[p_{n l} \frac{d q_{n l}}{d \rho}-q_{n l} \frac{d p_{n l}}{d \rho}\right]=\frac{-1}{r^{2} f^{1 / 2}} .
$$

Above, it is assumed that the field is in a nonzero temperature vacuum state defined with respect to the timelike Killing vector.

By the change of variables

$$
\begin{aligned}
& p_{n l}=\frac{1}{\sqrt{2 r^{2} W}} \exp \left\{\int^{\rho} W f^{-1 / 2} d \rho\right\}, \\
& q_{n l}=\frac{1}{\sqrt{2 r^{2} W}} \exp \left\{-\int^{\rho} W f^{-1 / 2} d \rho\right\},
\end{aligned}
$$

one sees that the Wronskian condition (8) is satisfied by

$$
C_{n l}=1
$$

and the mode equation (7) gives the following equation for $W(\rho)$ :

$$
W^{2}=(2 \pi n T)^{2}+\frac{f}{r^{2}}\left[l(l+1)+2 \xi+m^{2} r^{2}\right]+\frac{f^{\prime}}{8} \frac{\left(W^{2}\right)^{\prime}}{W^{2}}+\frac{f}{4} \frac{\left(W^{2}\right)^{\prime \prime}}{W^{2}}-\frac{5 f}{16} \frac{\left(W^{2}\right)^{\prime 2}}{W^{4}}+V
$$

where

$$
V=f\left(\frac{\left(r^{2}\right)^{\prime \prime}}{2 r^{2}}+\frac{f^{\prime}\left(r^{2}\right)^{\prime}}{4 f r^{2}}-\frac{\left(r^{2}\right)^{\prime 2}}{4 r^{4}}\right)+\xi f\left(-\frac{f^{\prime \prime}}{f}-2 \frac{\left(r^{2}\right)^{\prime \prime}}{r^{2}}+\frac{f^{\prime 2}}{2 f^{2}}+\frac{\left(r^{2}\right)^{\prime 2}}{2 r^{4}}-\frac{f^{\prime}\left(r^{2}\right)^{\prime}}{f r^{2}}\right)
$$

The prime denotes a derivative with respect to $\rho$. 
Now one can write expression for $\left\langle\varphi^{2}(x, \tilde{x})\right\rangle_{\text {unren }}=G_{E}(x, \tilde{x})$ using expressions (6) (9) , and then suppose $\rho=\tilde{\rho}, \theta=\tilde{\theta}, \phi=\tilde{\phi}\left(P_{l}(1)=1\right)$. The superficial divergences in the sums over $l$ that appear in this case can be removed as in Refs. [10, 11, 32 34]:

$$
\begin{aligned}
\left\langle\varphi^{2}\right\rangle_{\text {unren }}= & \frac{T}{4 \pi r \sqrt{f}} \sum_{l=0}^{\infty}\left[\sqrt{\frac{f}{r^{2}}} \frac{(l+1 / 2)}{W_{\mid n=0}}-1\right] \\
& +\frac{T}{2 \pi r \sqrt{f}} \sum_{n=1}^{\infty} \cos \left(u_{n} \varepsilon\right) \sum_{l=0}^{\infty}\left[\sqrt{\frac{f}{r^{2}}} \frac{(l+1 / 2)}{W}-1\right], \\
\varepsilon & =\sqrt{\frac{f}{r^{2}}}(\tau-\tilde{\tau}), \quad u_{n}=2 \pi n T \sqrt{\frac{r^{2}}{f}} .
\end{aligned}
$$

\section{WKB APPROXIMATION}

Equation (11) can be solved iteratively when the metric functions $f(\rho)$ and $r^{2}(\rho)$ are varying slowly, that is,

$$
\varepsilon_{W K B}=L_{\star} / L \ll 1,
$$

where

$$
L_{\star}(\rho)=\frac{r(\rho)}{\sqrt{\left|2 \xi+m^{2} r^{2}(\rho)\right|}},
$$

and $L$ is a characteristic scale of variation of the metric functions:

$$
\frac{1}{L(\rho)}=\max \left\{\left|\frac{r^{\prime}}{r}\right|,\left|\frac{f^{\prime}}{f}\right|,\left|\frac{r^{\prime}}{r} \sqrt{|\xi|}\right|,\left|\frac{f^{\prime}}{f} \sqrt{|\xi|}\right|,\left|\frac{r^{\prime \prime}}{r}\right|^{1 / 2},\left|\frac{f^{\prime \prime}}{f}\right|^{1 / 2}, \ldots\right\} .
$$

We shall call the region of spacetime where the metric functions $f(\rho)$ and $r^{2}(\rho)$ is slowly varying the long throat. This type of region exists, for example, in the neighborhood of the ultraextremal horizon [35].

The zeroth-order WKB solution of Eq. (11) corresponds to neglecting terms with derivatives in this equation

$$
W^{2}=U_{0},
$$

where

$$
\begin{aligned}
U_{0} & =(2 \pi n T)^{2}+\frac{f}{r^{2}}\left(l+\frac{1}{2}\right)^{2}+\frac{f}{r^{2}} \mu^{2}, \\
\mu^{2} & =m^{2} r^{2}+2 \xi-\frac{1}{4}
\end{aligned}
$$


Let us stress that $U_{0}$ is the exact solution of Eq. (11) in a spacetime with metric $d s^{2}=$ $f_{0} d \tau^{2}+d \rho^{2}+r_{0}^{2}\left(d \theta^{2}+\sin ^{2} \theta d \varphi^{2}\right)$, where $f_{0}$ and $r_{0}$ are constants.

Below as in Ref. [36] it is assumed that

$$
\mu^{2}>0 .
$$

The forth-order solution is

$$
W^{2}=U_{0}+U_{2}+U_{4}
$$

with

$$
U_{2}=V+\frac{f^{\prime}}{8} \frac{U_{0}{ }^{\prime}}{U_{0}}+\frac{f}{4} \frac{U_{0}^{\prime \prime}}{U_{0}}-\frac{5 f}{16} \frac{U_{0}^{\prime 2}}{U_{0}{ }^{2}}
$$

and

$$
\begin{aligned}
& U_{4}=\frac{f^{\prime}}{8}\left(\frac{U_{2}{ }^{\prime}}{U_{0}}-\frac{U_{0}{ }^{\prime} U_{2}}{U_{0}{ }^{2}}\right)+\frac{f}{4}\left(\frac{U_{2}^{\prime \prime}}{U_{0}}-\frac{U_{0}{ }^{\prime \prime} U_{2}}{U_{0}{ }^{2}}\right)-\frac{5 f}{8}\left(\frac{U_{0}{ }^{\prime} U_{2}{ }^{\prime}}{U_{0}{ }^{2}}-\frac{U_{0}{ }^{2} U_{2}}{U_{0}{ }^{3}}\right) \\
& =\frac{1}{U_{0}}\left[\frac{f^{\prime} V^{\prime}}{8}+\frac{f V^{\prime \prime}}{4}\right]+\frac{1}{U_{0}^{2}}\left[\left(\frac{f^{\prime} f^{\prime \prime}}{64}-\frac{5 f V^{\prime}}{8}+\frac{f f^{\prime \prime \prime}}{32}-\frac{f^{\prime} V}{8}\right) U_{0}^{\prime}\right. \\
& \left.+\left(\frac{f f^{\prime \prime}}{8}+\frac{3 f^{\prime 2}}{64}-\frac{f V}{4}\right) U_{0}^{\prime \prime}+\frac{3 f f^{\prime}}{16} U_{0}^{\prime \prime \prime}+\frac{f^{2}}{16} U_{0}^{\prime \prime \prime \prime}\right] \\
& +\frac{1}{U_{0}^{3}}\left[\left(-\frac{9 f^{\prime 2}}{128}-\frac{7 f f^{\prime \prime}}{32}+\frac{5 f V}{8}\right){U_{0}{ }^{2}}^{2}-\frac{15 f f^{\prime}}{16} U_{0}{ }^{\prime} U_{0}{ }^{\prime \prime}-\frac{9 f^{2}}{32} U_{0}{ }^{\prime \prime 2}\right. \\
& \left.-\frac{7 f^{2}}{16} U_{0}^{\prime} U_{0}^{\prime \prime \prime}\right]+\frac{1}{U_{0}{ }^{4}}\left[\frac{27 f f^{\prime}}{32} U_{0}{ }^{\prime 3}+\frac{27 f^{2}}{16}{U_{0}^{\prime}}^{\prime 2} U_{0}{ }^{\prime \prime}\right]-\frac{1}{U_{0}{ }^{5}}\left[\frac{135 f^{2}}{128} U_{0}{ }^{4}\right] .
\end{aligned}
$$

The approximate (of fourth WKB order) expression for $\left\langle\varphi^{2}\right\rangle_{\text {unren }}$ is obtained by substituting the WKB expansion of $W^{2}$ [Eq. (21)] into Eq. (13)):

$$
\begin{aligned}
& \left\langle\varphi^{2}\right\rangle_{\text {unren }}=\frac{1}{4 \pi^{2}}\left\{\frac{1}{r^{2}} \mathbf{S}_{\mathbf{0}}^{\mathbf{0}}(\varepsilon, \mu)-\frac{V}{2 f} \mathbf{S}_{\mathbf{1}}^{\mathbf{0}}(\varepsilon, \mu)-\frac{r^{2}}{16 f^{2}}\left[f^{\prime}\left(\frac{f}{r^{2}}\right)^{\prime}+2 f\left(\frac{f}{r^{2}}\right)^{\prime \prime}\right] \mathbf{S}_{\mathbf{2}}^{\mathbf{1}}(\varepsilon, \mu)\right. \\
& -\frac{r^{2}}{16 f^{2}}\left[-6 V^{2}+2 f\left(\mu^{2} \frac{f}{r^{2}}\right)^{\prime \prime}+f^{\prime} V^{\prime}+f^{\prime}\left(\mu^{2} \frac{f}{r^{2}}\right)^{\prime}+2 f V^{\prime \prime}\right] \mathbf{S}_{\mathbf{2}}^{\mathbf{0}}(\varepsilon, \mu) \\
& +\frac{5 r^{4}}{32 f^{2}}\left(\frac{f}{r^{2}}\right)^{\prime 2} \mathbf{S}_{\mathbf{3}}^{2}(\varepsilon, \mu)-\frac{r^{4}}{128 f^{3}}\left[f^{\prime} f^{\prime \prime}\left(\frac{f}{r^{2}}\right)^{\prime}+8 f f^{\prime \prime}\left(\frac{f}{r^{2}}\right)^{\prime \prime}-40 f V^{\prime}\left(\frac{f}{r^{2}}\right)^{\prime}\right. \\
& +3 f^{\prime 2}\left(\frac{f}{r^{2}}\right)^{\prime \prime}+12 f^{\prime} f\left(\frac{f}{r^{2}}\right)^{\prime \prime \prime}-40 f\left(\frac{f}{r^{2}}\right)^{\prime}\left(\mu^{2} \frac{f}{r^{2}}\right)^{\prime}+2 f f^{\prime \prime \prime}\left(\frac{f}{r^{2}}\right)^{\prime}-20 V f^{\prime}\left(\frac{f}{r^{2}}\right)^{\prime} \\
& \left.+4(f)^{2}\left(\frac{f}{r^{2}}\right)^{\prime \prime \prime \prime}-40 V f\left(\frac{f}{r^{2}}\right)^{\prime \prime}\right] \mathbf{S}_{\mathbf{3}}^{\mathbf{1}}(\varepsilon, \mu)-\frac{r^{4}}{128 f^{3}}\left[4 f^{2}\left(\mu^{2} \frac{f}{r^{2}}\right)^{\prime \prime \prime \prime \prime}+f^{\prime} f^{\prime \prime}\left(\mu^{2} \frac{f}{r^{2}}\right)^{\prime}\right.
\end{aligned}
$$




$$
\begin{aligned}
& -40 f V^{\prime}\left(\mu^{2} \frac{f}{r^{2}}\right)^{\prime}-20 V f^{\prime}\left(\mu^{2} \frac{f}{r^{2}}\right)^{\prime}+8 f f^{\prime \prime}\left(\mu^{2} \frac{f}{r^{2}}\right)^{\prime \prime}-20 f\left(\mu^{2} \frac{f}{r^{2}}\right)^{\prime 2} \\
& \left.-40 V f\left(\mu^{2} \frac{f}{r^{2}}\right)^{\prime \prime}+3 f^{\prime 2}\left(\mu^{2} \frac{f}{r^{2}}\right)^{\prime \prime}+12 f^{\prime} f\left(\mu^{2} \frac{f}{r^{2}}\right)^{\prime \prime \prime}+2 f f^{\prime \prime \prime}\left(\mu^{2} \frac{f}{r^{2}}\right)^{\prime}\right] \mathbf{S}_{\mathbf{3}}^{\mathbf{0}}(\varepsilon, \mu) \\
& -\frac{r^{6}}{512 f^{4}}\left[-84 f^{2}\left(\frac{f}{r^{2}}\right)^{\prime \prime 2}-112 f^{2}\left(\frac{f}{r^{2}}\right)^{\prime \prime \prime}\left(\frac{f}{r^{2}}\right)^{\prime}-21 f^{\prime 2}\left(\frac{f}{r^{2}}\right)^{\prime 2}-56 f f^{\prime \prime}\left(\frac{f}{r^{2}}\right)^{\prime 2}\right. \\
& \left.+280 V f\left(\frac{f}{r^{2}}\right)^{\prime 2}-252 f^{\prime} f\left(\frac{f}{r^{2}}\right)^{\prime}\left(\frac{f}{r^{2}}\right)^{\prime \prime}\right] \mathbf{S}_{\mathbf{4}}^{2}(\varepsilon, \mu)-\frac{r^{6}}{256 f^{4}}\left[-56 f f^{\prime \prime}\left(\frac{f}{r^{2}}\right)^{\prime}\left(\mu^{2} \frac{f}{r^{2}}\right)^{\prime}\right. \\
& -21 f^{\prime 2}\left(\frac{f}{r^{2}}\right)^{\prime}\left(\mu^{2} \frac{f}{r^{2}}\right)^{\prime}+280 V f\left(\frac{f}{r^{2}}\right)^{\prime}\left(\mu^{2} \frac{f}{r^{2}}\right)^{\prime}-56 f^{2}\left(\mu^{2} \frac{f}{r^{2}}\right)^{\prime \prime \prime}\left(\frac{f}{r^{2}}\right)^{\prime} \\
& -126 f^{\prime} f\left(\mu^{2} \frac{f}{r^{2}}\right)^{\prime}\left(\frac{f}{r^{2}}\right)^{\prime \prime}-84(f)^{2}\left(\frac{f}{r^{2}}\right)^{\prime \prime}\left(\mu^{2} \frac{f}{r^{2}}\right)^{\prime \prime}-126 f^{\prime} f\left(\frac{f}{r^{2}}\right)^{\prime}\left(\mu^{2} \frac{f}{r^{2}}\right)^{\prime \prime} \\
& \left.-56(f)^{2}\left(\frac{f}{r^{2}}\right)^{\prime \prime \prime}\left(\mu^{2} \frac{f}{r^{2}}\right)^{\prime}\right] \mathbf{S}_{\mathbf{4}}^{\mathbf{1}}(\varepsilon, \mu)-\frac{r^{6}}{512 f^{4}}\left[280 V f\left(\mu^{2} \frac{f}{r^{2}}\right)^{\prime 2}\left(\frac{f}{r^{2}}\right)^{2}\right. \\
& -112 f^{2}\left(\mu^{2} \frac{f}{r^{2}}\right)^{\prime}\left(\mu^{2} \frac{f}{r^{2}}\right)^{\prime \prime \prime}\left(\frac{f}{r^{2}}\right)^{2}-252 f^{\prime} f\left(\mu^{2} \frac{f}{r^{2}}\right)^{\prime}\left(\mu^{2} \frac{f}{r^{2}}\right)^{\prime \prime}\left(\frac{f}{r^{2}}\right)^{2} \\
& \left.-21 f^{\prime 2}\left(\mu^{2} \frac{f}{r^{2}}\right)^{\prime 2}\left(\frac{f}{r^{2}}\right)^{2}-56 f f^{\prime \prime}\left(\mu^{2} \frac{f}{r^{2}}\right)^{\prime 2}\left(\frac{f}{r^{2}}\right)^{2}-84 f^{2}\left(\mu^{2} \frac{f}{r^{2}}\right)^{\prime \prime 2}\left(\frac{f}{r^{2}}\right)^{2}\right] \mathbf{S}_{4}^{0}(\varepsilon, \mu) \\
& -\frac{231 r^{8}}{512 f^{4}}\left[2 f\left(\frac{f}{r^{2}}\right)^{\prime 2}\left(\frac{f}{r^{2}}\right)^{\prime \prime}+f\left(\frac{f}{r^{2}}\right)^{\prime 3} f^{\prime}\right] \mathbf{S}_{\mathbf{5}}^{\mathbf{3}}(\varepsilon, \mu)-\frac{231 r^{8}}{512 f^{4}}\left[3 f^{\prime}\left(\frac{f}{r^{2}}\right)^{\prime 2}\left(\mu^{2} \frac{f}{r^{2}}\right)^{\prime}\right. \\
& \left.+4 f\left(\frac{f}{r^{2}}\right)^{\prime \prime}\left(\frac{f}{r^{2}}\right)^{\prime}\left(\mu^{2} \frac{f}{r^{2}}\right)^{\prime}+2 f\left(\mu^{2} \frac{f}{r^{2}}\right)^{\prime \prime}\left(\frac{f}{r^{2}}\right)^{\prime 2}\right] \mathbf{S}_{\mathbf{5}}^{\mathbf{2}}(\varepsilon, \mu) \\
& -\frac{231 r^{8}}{512 f^{4}}\left[2 f\left(\frac{f}{r^{2}}\right)^{\prime \prime}\left(\mu^{2} \frac{f}{r^{2}}\right)^{\prime 2}+4 f\left(\mu^{2} \frac{f}{r^{2}}\right)^{\prime \prime}\left(\frac{f}{r^{2}}\right)^{\prime}\left(\mu^{2} \frac{f}{r^{2}}\right)^{\prime}\right. \\
& \left.+3 f^{\prime}\left(\frac{f}{r^{2}}\right)^{\prime}\left(\mu^{2} \frac{f}{r^{2}}\right)^{\prime 2}\right] \mathbf{S}_{\mathbf{5}}^{\mathbf{1}}(\varepsilon, \mu)-\frac{231 r^{8}}{512 f^{4}}\left[2 f\left(\mu^{2} \frac{f}{r^{2}}\right)^{\prime \prime}\left(\mu^{2} \frac{f}{r^{2}}\right)^{\prime 2}\right. \\
& \left.+f^{\prime}\left(\mu^{2} \frac{f}{r^{2}}\right)^{\prime 3}\right] \mathbf{S}_{\mathbf{5}}^{\mathbf{0}}(\varepsilon, \mu)+\frac{1155 r^{10}}{2048 f^{4}}\left(\frac{f}{r^{2}}\right)^{\prime 4} \mathbf{S}_{\mathbf{6}}^{\mathbf{4}}(\varepsilon, \mu) \\
& +\frac{1155 r^{10}}{512 f^{4}}\left(\frac{f}{r^{2}}\right)^{\prime 3}\left(\mu^{2} \frac{f}{r^{2}}\right)^{\prime} \mathbf{S}_{\mathbf{6}}^{\mathbf{3}}(\varepsilon, \mu)+\frac{3465 r^{10}}{1024 f^{4}}\left(\frac{f}{r^{2}}\right)^{\prime 2}\left(\mu^{2} \frac{f}{r^{2}}\right)^{\prime 2} \mathbf{S}_{\mathbf{6}}^{2}(\varepsilon, \mu) \\
& \left.+\frac{1155 r^{10}}{512 f^{4}}\left(\frac{f}{r^{2}}\right)^{\prime}\left(\mu^{2} \frac{f}{r^{2}}\right)^{\prime 3} \mathbf{S}_{\mathbf{6}}^{\mathbf{1}}(\varepsilon, \mu)+\frac{1155 r^{10}}{2048 f^{4}}\left(\mu^{2} \frac{f}{r^{2}}\right)^{\prime 4} \mathbf{S}_{\mathbf{6}}^{\mathbf{0}}(\varepsilon, \mu)\right\}
\end{aligned}
$$


The mode sums and integrals in these expressions are of the form

$$
\begin{aligned}
& S_{k}^{m}(\varepsilon, \mu)=\frac{\pi T r}{\sqrt{f}} \sum_{l=0}^{\infty}\left\{\frac{(l+1 / 2)^{2 m+1}}{\left[\mu^{2}+(l+1 / 2)^{2}\right]^{(2 k+1) / 2}}-\text { subtraction terms }\right\} \\
& +\frac{2 \pi T r}{\sqrt{f}} \sum_{n=1}^{\infty} \cos \left(\varepsilon u_{n}\right) \sum_{l=0}^{\infty}\left\{\frac{(l+1 / 2)^{2 m+1}}{\left[u_{n}^{2}+\mu^{2}+(l+1 / 2)^{2}\right]^{(2 k+1) / 2}}-\text { subtraction terms }\right\},
\end{aligned}
$$

where $m$ and $k$ are integers and $m, k \geq 0$. The subtraction terms for the sum over $l$ can be obtained by expanding the function that is summed in inverse powers of $l$ and truncating at $O\left(l^{0}\right)$. Such subtracting corresponds to removing the superficial divergences in the sums over $l$ discussed above:

$$
\begin{aligned}
S_{m}^{m}(\varepsilon, \mu)= & \frac{\pi T r}{\sqrt{f}} \sum_{l=0}^{\infty}\left\{\frac{(l+1 / 2)^{2 m+1}}{\left[\mu^{2}+(l+1 / 2)^{2}\right]^{(2 m+1) / 2}}-1\right\} \\
& +\frac{2 \pi T r}{\sqrt{f}} \sum_{n=1}^{\infty} \cos \left(\varepsilon u_{n}\right) \sum_{l=0}^{\infty}\left\{\frac{(l+1 / 2)^{2 m+1}}{\left[u_{n}^{2}+\mu^{2}+(l+1 / 2)^{2}\right]^{(2 m+1) / 2}}-1\right\} .
\end{aligned}
$$

For other quantities $S_{k}^{m}(\varepsilon, \mu)$ there are no subtraction terms. The details of calculations of $S_{k}^{m}(\varepsilon, \mu)$ in the limit $\varepsilon \rightarrow 0$ are discussed in Appendix A:

$$
\begin{aligned}
& S_{0}^{0}(\varepsilon, \mu)=\frac{1}{\varepsilon^{2}}+4 \mu^{2} J(a / \mu)+\left(\frac{\mu^{2}}{2}-\frac{1}{24}\right)\left(C+\frac{1}{2} \ln \left|\frac{\mu^{2} \varepsilon^{2}}{4}\right|\right) \\
& -\frac{\mu^{2}}{4}-\mu^{2} I_{1}(\mu)+O\left(\varepsilon^{2} \ln |\varepsilon|\right), \\
& S_{1}^{0}(\varepsilon, \mu)=-\left(C+\frac{1}{2} \ln \left|\frac{\mu^{2} \varepsilon^{2}}{4}\right|\right)+\mu \frac{d}{d \mu} I_{0}(\mu)+O\left(\varepsilon^{2} \ln |\varepsilon|\right), \\
& S_{m+1}^{m}(\varepsilon, \mu)=\frac{1}{(2 m+1) ! !}\left[-(2 m) ! !\left(C+\frac{1}{2} \ln \left|\frac{\mu^{2} \varepsilon^{2}}{4}\right|\right)+\mu^{2}\left(\frac{\partial}{\mu \partial \mu}\right)^{m+1}\left(\mu^{2 m} I_{0}(\mu)\right)\right] \\
& +O\left(\varepsilon^{2} \ln |\varepsilon|\right) \quad(m \geq 1), \\
& S_{k}^{m}(\varepsilon, \mu)=\frac{(2 m+1) ! !}{(2 k+1) ! !}\left(-\frac{\partial}{\mu \partial \mu}\right)^{k-m-1} S_{m+1}^{m}(\varepsilon, \mu) \quad(m \geq 0, k \geq m+2),
\end{aligned}
$$

where

$$
J(a / \mu)=\int_{1}^{\infty} \frac{\sqrt{\eta^{2}-1}}{e^{2 \pi \mu \eta / a}-1} d \eta, \quad I_{m}(\mu)=\int_{0}^{\infty} \frac{\eta^{2 m-1} \ln \left|1-\eta^{2}\right|}{1+e^{2 \pi \mu \eta}} d \eta
$$


The renormalization of $\left\langle\varphi^{2}\right\rangle$ is achieved by subtracting the renormalization counterterm from $\left\langle\varphi^{2}\right\rangle_{\text {unren }}$ and then letting $\tilde{\tau} \rightarrow \tau:$

$$
\left\langle\varphi^{2}\right\rangle_{r e n}=\lim _{\tilde{\tau} \rightarrow \tau}\left[\left\langle\varphi^{2}\right\rangle_{\text {unren }}-\left\langle\varphi^{2}\right\rangle_{\mathrm{DS}}\right]
$$

where

$$
\begin{aligned}
\left\langle\phi^{2}\right\rangle_{\mathrm{DS}}= & \frac{1}{8 \pi^{2} \sigma}+\frac{1}{8 \pi^{2}}\left[m^{2}+\left(\xi-\frac{1}{6}\right) R\right]\left[C+\frac{1}{2} \ln \left(\frac{m_{D S}^{2}|\sigma|}{2}\right)\right] \\
& -\frac{m^{2}}{16 \pi^{2}}+\frac{1}{96 \pi^{2}} R_{\alpha \beta} \frac{\sigma^{\alpha} \sigma^{\beta}}{\sigma}
\end{aligned}
$$

$C$ is Euler's constant, $R_{\alpha \beta}$ is the Ricci tensor, $R=R_{\alpha}^{\alpha}, \sigma$ is one-half the square of the distance between the points $x$ and $\tilde{x}$ along the shortest geodesic connecting them, $\sigma^{\mu}$ is the covariant derivative of $\sigma$ [37].

$$
\begin{aligned}
& \sigma^{i}=-\left(x^{i}-\tilde{x}^{i}\right)-\frac{1}{2} \Gamma_{j k}^{i}\left(x^{j}-\tilde{x}^{j}\right)\left(x^{k}-\tilde{x}^{k}\right) \\
&-\frac{1}{6}\left(\Gamma_{j m}^{i} \Gamma_{k l}^{m}+\frac{\partial \Gamma_{j k}^{i}}{\partial \tilde{x}^{l}}\right)\left(x^{j}-\tilde{x}^{j}\right)\left(x^{k}-\tilde{x}^{k}\right)\left(x^{l}-\tilde{x}^{l}\right)+O\left((x-\tilde{x})^{4}\right), \\
& \sigma=\frac{1}{2} g_{\mu \nu} \sigma^{\mu} \sigma^{\nu}
\end{aligned}
$$

where Christoffel symbols $\Gamma_{j k}^{i}$ are calculated at point $\tilde{x}$. For the metric (44) the quantities $\sigma^{\mu}$ and $\sigma$ are

$$
\begin{aligned}
\sigma^{\tau} & =-(\tau-\tilde{\tau})+\frac{f^{\prime 2}}{24 f}(\tau-\tilde{\tau})^{3}+O\left((\tau-\tilde{\tau})^{5}\right), \\
\sigma^{\rho} & =\frac{f^{\prime}}{4}(\tau-\tilde{\tau})^{2}+O\left((\tau-\tilde{\tau})^{4}\right), \\
\sigma^{\theta} & =\sigma^{\phi}=0 .
\end{aligned}
$$

The constant $m_{D S}$ is equal to the mass $m$ of the field for a massive scalar field. For a massless field $m_{D S}$ is an arbitrary parameter due to the infrared cutoff in $\left\langle\varphi^{2}\right\rangle_{\mathrm{DS}}$. A particular choice of the value of $m_{D S}$ corresponds to a finite renormalization of the coefficients of terms in the gravitational Lagrangian and must be fixed by experiment or observation.

$$
\begin{aligned}
\left\langle\phi^{2}\right\rangle_{\mathrm{DS}}= & \frac{1}{8 \pi^{2}}\left\{\frac{2}{f(\tau-\tilde{\tau})^{2}}+\frac{f^{\prime 2}}{f^{2}}+\left[m^{2}+\left(\xi-\frac{1}{6}\right)\left(\frac{2}{r^{2}}-\frac{f^{\prime \prime}}{f}-\frac{2 r^{2^{\prime \prime}}}{r^{2}}+\frac{f^{\prime 2}}{2 f^{2}}-\frac{f^{\prime} r^{2^{\prime}}}{f r^{2}}\right.\right.\right. \\
& \left.\left.\left.+\frac{r^{2^{2}}}{2 r^{4}}\right)\right]\left[C+\frac{1}{2} \ln \left(\frac{m_{D S}^{2} f(\tau-\tilde{\tau})^{2}}{4}\right)\right]-\frac{m^{2}}{2}-\frac{f^{\prime} r^{2^{\prime}}}{12 f r^{2}}-\frac{f^{\prime \prime}}{12 f}\right\} .
\end{aligned}
$$


The procedure described above gives renormalized expression for $\left\langle\varphi^{2}\right\rangle_{\text {ren }}$ in the framework of the second-order WKB approximation:

$$
\begin{aligned}
& 4 \pi^{2}\left\langle\varphi^{2}\right\rangle_{r e n}=4 \pi^{2}\left(\left\langle\varphi^{2}\right\rangle_{r e n}^{(0)}+\left\langle\varphi^{2}\right\rangle_{r e n}^{(2)}\right) \\
& =\frac{4 \mu^{2}}{r^{2}} J(a / \mu)-\frac{1}{4 r^{2}}\left(2 \xi-\frac{1}{4}\right)+\frac{1}{4 r^{2}}\left[m^{2} r^{2}+2\left(\xi-\frac{1}{6}\right)\right] \ln \left|\frac{m^{2} r^{2}+2 \xi-1 / 4}{m_{D S}^{2} r^{2}}\right| \\
& -\frac{\mu^{2}}{r^{2}} I_{1}(\mu)+\frac{r^{2^{\prime \prime}}}{24 r^{2}}-\frac{r^{2^{\prime 2}}}{48 r^{4}}+\frac{f^{\prime} r^{2^{\prime}}}{48 f r^{2}}+\left[\frac{f^{\prime \prime}}{12 f}+\frac{r^{2^{\prime \prime}}}{6 r^{2}}-\frac{f^{\prime 2}}{24 f^{2}}-\frac{r^{2^{2}}}{24 r^{4}}+\frac{f^{\prime} r^{2^{\prime}}}{12 f r^{2}}\right. \\
& \left.+\xi\left(-\frac{f^{\prime \prime}}{2 f}-\frac{r^{2^{\prime \prime}}}{r^{2}}+\frac{f^{\prime 2}}{4 f^{2}}+\frac{r^{2^{\prime 2}}}{4 r^{4}}-\frac{f^{\prime} r^{2^{\prime}}}{2 f r^{2}}\right)\right]\left(\frac{1}{2} \ln \left|\frac{m^{2} r^{2}+2 \xi-1 / 4}{m_{D S}^{2} r^{2}}\right|-\mu \frac{d I_{0}(\mu)}{d \mu}\right),
\end{aligned}
$$

where $a=2 \pi \operatorname{Tr} / \sqrt{f}$. The terms of a fourth WKB order are given in Appendix B.

\section{CONCLUSION}

We have obtained an analytical approximation for $\left\langle\varphi^{2}\right\rangle_{\text {ren }}$ of quantized scalar fields in the background of a long throat (15)-(17). The field is assumed to be both massive or massless, with arbitrary coupling to the scalar curvature, and in a thermal state at an arbitrary temperature.

As an example, we consider spacetime with metric

$$
d s^{2}=-f(r) d t^{2}+\frac{d r^{2}}{f(r)}+r^{2}\left(d \theta^{2}+\sin ^{2} \theta d \varphi^{2}\right)
$$

where $f(r)$ is

$$
f(r)=-\frac{\left(r+3 r_{+}\right)}{6 r_{+}^{2} r^{2}}\left(r-r_{+}\right)^{3} .
$$

It was shown in [35] the WKB approximation (15)-(17) remains valid near ultraextremal horizon $r=r_{+}$It means that we can obtain explicit expression for $\left\langle\varphi^{2}\right\rangle_{r e n}$ near ultraextremal 
horizon $r=r_{+}$

$$
\begin{aligned}
& 4 \pi^{2}\left\langle\varphi^{2}\right\rangle_{\text {ren }}=\frac{4 \mu^{2}}{r_{+}^{2}} J\left(a / \mu_{+}\right)-\frac{1}{4 r_{+}^{2}}\left(2 \xi-\frac{1}{4}\right)+\frac{1}{4 r_{+}^{2}}\left[m^{2} r_{+}^{2}\right. \\
& \left.+2\left(\xi-\frac{1}{6}\right)\right] \ln \left|\frac{\mu_{+}^{2}}{m_{D S}^{2} r^{2}}\right|-\frac{\mu_{+}^{2}}{r_{+}^{2}} I_{1}\left(\mu_{+}\right)+\left[\frac{2 \xi-1 / 4}{24 r_{+}^{3} \mu_{+}^{2}}+2 \frac{2 \xi-1 / 4}{r_{+}^{3}} I_{1}\left(\mu_{+}\right)\right. \\
& \left.+\frac{m^{2} \mu_{+}}{r_{+}} \frac{d I_{1}\left(\mu_{+}\right)}{d \mu_{+}}+\frac{2 \mu_{+}}{r_{+}^{3}}\left(\xi-\frac{1}{6}\right) \frac{d I_{0}\left(\mu_{+}\right)}{d \mu_{+}}\right]\left(r-r_{+}\right) \\
& +\left[\frac{-128 m^{4} r_{+}^{4}+52 m^{2} r_{+}^{2}-3-480 m^{2} r_{+}^{2} \xi+64 \xi-320 \xi^{2}}{192 r_{+}^{4} \mu_{+}^{4}}\right. \\
& -3 \frac{2 \xi-1 / 4}{r_{+}^{4}} I_{1}\left(\mu_{+}\right)+\frac{3 m^{2}(2 \xi-1 / 4)}{2 \mu_{+} r_{+}^{2}} \frac{d I_{1}\left(\mu_{+}\right)}{d \mu_{+}}-\frac{m^{4}}{2} \frac{d^{2} I_{1}\left(\mu_{+}\right)}{d \mu_{+}^{2}} \\
& +\frac{4 m^{2} r_{+}^{2}-3-24 m^{2} r_{+}^{2} \xi+50 \xi-192 \xi^{2}}{24 r_{+}^{4} \mu_{+}} \frac{d I_{0}\left(\mu_{+}\right)}{d \mu_{+}} \\
& \left.+\frac{-m^{2} r_{+}^{2}+6 m^{2} r_{+}^{2} \xi+\xi-6 \xi^{2}}{3 r_{+}^{4}} \frac{d^{2} I_{0}\left(\mu_{+}\right)}{d \mu_{+}^{2}}\right]\left(r-r_{+}\right)^{2}+O\left(\left(r-r_{+}\right)^{3}\right),
\end{aligned}
$$

where $a_{+}=\frac{2 \pi T r_{+}}{\sqrt{f\left(r_{+}\right)}}, \mu_{+}^{2}=m^{2} r_{+}^{2}+2 \xi-1 / 4$.

\section{Acknowledgments}

The work is performed according to the Russian Government Program of Competitive Growth of Kazan Federal University.

\section{Appendix A}

To calculate the quantities $S_{k}^{m}(\varepsilon, \mu)$ it is necessary to compute the various sums over $l$. We start from the sums in expression (25):

$$
\begin{aligned}
& S_{k}^{m}(\varepsilon, \mu)=\frac{\pi T r}{\sqrt{f}} \sum_{l=0}^{\infty}\left\{\frac{(l+1 / 2)^{2 m+1}}{\left[\mu^{2}+(l+1 / 2)^{2}\right]^{(2 k+1) / 2}}-\text { subtraction terms }\right\} \\
& +\frac{2 \pi T r}{\sqrt{f}} \sum_{n=1}^{\infty} \cos \left(\varepsilon u_{n}\right) \sum_{l=0}^{\infty}\left\{\frac{(l+1 / 2)^{2 m+1}}{\left[u_{n}^{2}+\mu^{2}+(l+1 / 2)^{2}\right]^{(2 k+1) / 2}}-\text { subtraction terms }\right\},
\end{aligned}
$$

For calculation of this sum we can use the Abel-Plana method [38-40]

$$
\sum_{l=0}^{\infty} F(l+1 / 2)=\int_{q}^{\infty} F(x) d x+\int_{q-i \infty}^{q} \frac{F(z)}{1+e^{i 2 \pi z}} d z-\int_{q}^{q+i \infty} \frac{F(z)}{1+e^{-i 2 \pi z}} d z,
$$


where $-1 / 2<q<1 / 2, f(z)$ is a holomorphic function for $\operatorname{Re} z \geq q, f(z)$ satisfies the condition

$$
|F(x+i y)|<\epsilon(x) e^{a|y|}, \quad 0<a<2 \pi
$$

and $\epsilon(x)$ is an arbitrary function with asymptotic behavior

$$
\epsilon(x) \rightarrow 0 \text { for } \quad x \rightarrow+\infty
$$

Using this formula we can calculate the sums in Eqs. (25):

$$
\begin{aligned}
& \sum_{l=0}^{\infty}\left\{\frac{(l+1 / 2)^{2 m+1}}{\left[u_{n}^{2}+\mu^{2}+(l+1 / 2)^{2}\right]^{(2 m+1) / 2}}-1\right\}=\lim _{q \rightarrow 0}\left\{\int_{q}^{\infty}\left[\frac{x^{2 m+1}}{\left(u_{n}^{2}+\mu^{2}+x^{2}\right)^{(2 m+1) / 2}}-1\right] d x\right. \\
& +\int_{q-i \infty}^{q}\left[\frac{z^{2 m+1}}{\left(u_{n}^{2}+\mu^{2}+z^{2}\right)^{(2 m+1) / 2}}-1\right] \frac{d z}{\left(1+e^{i 2 \pi z}\right)}-\int_{q}^{q+i \infty}\left[\frac{z^{2 m+1}}{\left(u_{n}^{2}+\mu^{2}+z^{2}\right)^{(2 m+1) / 2}}\right. \\
& \left.-1] \frac{d z}{\left(1+e^{-i 2 \pi z}\right)}\right\}=\sum_{k=0}^{m} \frac{(-1)^{m+k} m !}{k !(m-k) !} \frac{\sqrt{u_{n}^{2}+\mu^{2}}}{(2 m-2 k-1)} \\
& +2(-1)^{m} \lim _{\delta \rightarrow 0}\left[\int_{0}^{\sqrt{u_{n}^{2}+\mu^{2}}-\delta} \frac{x^{2 m+1}}{\left(1+e^{2 \pi x}\right)\left(u_{n}^{2}+\mu^{2}-x^{2}\right)^{(2 m+1) / 2}} d x\right. \\
& \left.-\left(\begin{array}{c}
\text { terms of this integral that } \\
\text { diverge in the limit } \delta \rightarrow 0
\end{array}\right)\right]=\sum_{i=0}^{m} \frac{(-1)^{m+i} m !}{i !(m-i) !} \frac{\sqrt{u_{n}^{2}+\mu^{2}}}{(2 m-2 i-1)} \\
& +\frac{2}{(2 m-1) ! !} \int_{0}^{\sqrt{u_{n}^{2}+\mu^{2}}} \frac{x d x}{\sqrt{u_{n}^{2}+\mu^{2}-x^{2}}}\left(\frac{d}{x d x}\right)^{m} \frac{x^{2 m}}{\left(1+e^{2 \pi x}\right)} \quad(m \geq 0) \text {, } \\
& \sum_{l=0}^{\infty} \frac{(l+1 / 2)^{2 m+1}}{\left[u_{n}^{2}+\mu^{2}+(l+1 / 2)^{2}\right]^{(2 m+3) / 2}}=\lim _{q \rightarrow 0}\left\{\int_{q}^{\infty} \frac{x^{2 m+1}}{\left(u_{n}^{2}+\mu^{2}+x^{2}\right)^{(2 m+3) / 2}} d x\right. \\
& \left.+\int_{q-i \infty}^{q} \frac{z^{2 m+1}}{\left(u_{n}^{2}+\mu^{2}+z^{2}\right)^{(2 m+3) / 2}} \frac{d z}{\left(1+e^{i 2 \pi z}\right)}-\int_{q}^{q+i \infty} \frac{z^{2 m+1}}{\left(u_{n}^{2}+\mu^{2}+z^{2}\right)^{(2 m+3) / 2}} \frac{d z}{\left(1+e^{-i 2 \pi z}\right)}\right\} \\
& =\frac{2^{m} m !}{(2 m+1) ! ! \sqrt{u_{n}^{2}+\mu^{2}}}+2(-1)^{m} \lim _{\delta \rightarrow 0}\left[\int_{0}^{\sqrt{u_{n}^{2}+\mu^{2}}-\delta} \frac{x^{2 m+1}}{\left(u_{n}^{2}+\mu^{2}-x^{2}\right)^{(2 m+3) / 2}} \frac{d x}{\left(1+e^{2 \pi x}\right)}\right. \\
& \left.-\left(\begin{array}{c}
\text { terms of this integral that } \\
\text { diverge in the limit } \delta \rightarrow 0
\end{array}\right)\right] \\
& =-\frac{2}{(2 m+1) ! !} \int_{0}^{\sqrt{u_{n}^{2}+\mu^{2}}} \frac{x d x}{\sqrt{u_{n}^{2}+\mu^{2}-x^{2}}}\left(\frac{d}{x d x}\right)^{m+1} \frac{x^{2 m}}{1+e^{2 \pi x}} \quad(m \geq 0),
\end{aligned}
$$




$$
\begin{aligned}
& \sum_{l=0}^{\infty} \frac{(l+1 / 2)^{2 m+1}}{\left[u_{n}^{2}+\mu^{2}+(l+1 / 2)^{2}\right]^{(2 k+1) / 2}} \\
& =\frac{(2 m+1) ! !}{(2 k-1) ! !}\left(-\frac{\partial}{\mu \partial \mu}\right)^{k-m-1} \sum_{l=0}^{\infty} \frac{(l+1 / 2)^{2 m+1}}{\left[u_{n}^{2}+\mu^{2}+(l+1 / 2)^{2}\right]^{(2 m+3) / 2}} \\
& \left.=\frac{2(-1)^{k-m}}{(2 k-1) ! !}\left(\frac{\partial}{\mu \partial \mu}\right) \int_{0}^{\sqrt{u_{n}^{2}+\mu^{2}}} \frac{x d x}{\sqrt{u_{n}^{2}+\mu^{2}-x^{2}}}\left(\frac{d}{x d x}\right)^{m+1} \frac{x^{2 m}}{1+e^{2 \pi x}} \quad\left(\begin{array}{c}
m \geq 0, \\
k \geq m+2
\end{array}\right) \mathrm{A} 7\right)
\end{aligned}
$$

Now we can calculate the sums over $n$ in (25).

$$
\begin{aligned}
\sum_{n=1}^{\infty} \sqrt{u_{n}^{2}+\mu^{2}} \cos \left(\varepsilon u_{n}\right) & =\left(-\frac{d^{2}}{d \varepsilon^{2}}+\mu^{2}\right) \sum_{n=1}^{\infty} \frac{\cos (\varepsilon a n)}{\sqrt{a^{2} n^{2}+\mu^{2}}} \\
& =a\left(-\frac{d^{2}}{d \tilde{\varepsilon}^{2}}+\tilde{\mu}^{2}\right)\left[-\frac{1}{\tilde{\mu}}+\sum_{n=0}^{\infty} \frac{\cos (\tilde{\varepsilon} n)}{\sqrt{n^{2}+\tilde{\mu}^{2}}}\right],
\end{aligned}
$$

where

$$
a=\frac{2 \pi T r}{\sqrt{f}}, \tilde{\varepsilon}=a \varepsilon, \tilde{\mu}=\frac{\mu}{a} .
$$

For calculation of the last sum over $n$ we can use the Plana sum formula [41]

$$
\sum_{n=0}^{\infty} F(n)=\frac{1}{2} F(0)+\lim _{q \rightarrow 0}\left\{\int_{q}^{\infty} F(y) d y+i \int_{q}^{\infty} \frac{d y}{e^{2 \pi y}-1}[F(q+i y)-F(q-i y)]\right\}
$$

Consequently

$$
\sum_{n=0}^{\infty} \frac{\cos (\tilde{\varepsilon} n)}{\sqrt{n^{2}+\tilde{\mu}^{2}}}=\frac{1}{2 \tilde{\mu}}+\int_{0}^{\infty} \frac{\cos (\tilde{\varepsilon} y)}{\sqrt{y^{2}+\tilde{\mu}^{2}}} d y+2 \int_{\tilde{\mu}}^{\infty} \frac{\cosh (\tilde{\varepsilon} y)}{\sqrt{y^{2}-\tilde{\mu}^{2}}} \frac{d y}{\left(e^{2 \pi y}-1\right)} .
$$

Note that the sum (A8) can be expanded in powers of $\varepsilon$ :

$$
\begin{aligned}
& \sum_{n=1}^{\infty} \sqrt{u_{n}^{2}+\mu^{2}} \cos \left(\varepsilon u_{n}\right)=a\left(-\frac{d^{2}}{d \tilde{\varepsilon}^{2}}+\tilde{\mu}^{2}\right)\left[-\frac{1}{2 \tilde{\mu}}+K_{0}(\tilde{\varepsilon} \tilde{\mu})\right. \\
& \left.+2 \int_{\tilde{\mu}}^{\infty} \frac{\cosh (\tilde{\varepsilon} y)}{\sqrt{y^{2}-\tilde{\mu}^{2}}} \frac{d y}{\left(e^{2 \pi y}-1\right)}\right]=a\left[-\frac{\tilde{\mu}}{2}-\frac{\tilde{\mu}}{\tilde{\varepsilon}} K_{1}(\tilde{\varepsilon} \tilde{\mu})\right. \\
& \left.-2 \int_{\tilde{\mu}}^{\infty} \frac{\cosh (\tilde{\varepsilon} y)}{\sqrt{y^{2}-\tilde{\mu}^{2}}} \frac{y^{2} d y}{\left(e^{2 \pi y}-1\right)}+2 \tilde{\mu}^{2} \int_{\tilde{\mu}}^{\infty} \frac{\cosh (\tilde{\varepsilon} y)}{\sqrt{y^{2}-\tilde{\mu}^{2}}} \frac{d y}{\left(e^{2 \pi y}-1\right)}\right] \\
& =a\left[-\frac{\tilde{\mu}}{2}-\frac{\tilde{\mu}^{2}}{2}\left(C+\frac{1}{2} \ln \frac{(\tilde{\varepsilon} \tilde{\mu})^{2}}{4}\right)-\frac{1}{\tilde{\varepsilon}^{2}}+\frac{\tilde{\mu}^{2}}{4}+O\left(\tilde{\varepsilon}^{2} \ln |\tilde{\varepsilon}|\right)\right] \\
& -2 a \int_{\tilde{\mu}}^{\infty} \frac{\sqrt{y^{2}-\tilde{\mu}^{2}} d y}{e^{2 \pi y}-1},
\end{aligned}
$$

where $K_{n}(x)$ is Macdonald's function and $C$ is Euler's constant. 
The second type of sum over $n$ has the form

$$
I_{-}=\sum_{n=1}^{\infty} \cos \left(\varepsilon u_{n}\right) \int_{0}^{\sqrt{u_{n}^{2}+\mu^{2}}} \frac{G(x)}{\sqrt{u_{n}^{2}+\mu^{2}-x^{2}}} d x .
$$

Using the formula (A10) one can obtain

$$
\begin{aligned}
& I_{-}=-\frac{1}{2} \int_{0}^{\tilde{\mu}} \frac{G(\tilde{x} a)}{\sqrt{\tilde{\mu}^{2}-\tilde{x}^{2}}} d \tilde{x}+\lim _{q \rightarrow 0}\left\{\int_{q}^{\infty} d y \cos (\tilde{\varepsilon} y) \int_{0}^{\sqrt{y^{2}+\tilde{\mu}^{2}}} \frac{G(\tilde{x} a)}{\sqrt{y^{2}+\tilde{\mu}^{2}-\tilde{x}^{2}}} d \tilde{x}\right. \\
& +i \int_{q}^{\infty} \frac{d y \cos [\tilde{\varepsilon}(q+i y)]}{e^{2 \pi y}-1} \int_{0}^{\sqrt{(q+i y)+\tilde{\mu}^{2}}} \frac{G(\tilde{x} a) d \tilde{x}}{\sqrt{(q+i y)^{2}+\tilde{\mu}^{2}-\tilde{x}^{2}}} \\
& \left.-i \int_{q}^{\infty} \frac{d y \cos [\tilde{\varepsilon}(q-i y)]}{e^{2 \pi y}-1} \int_{0}^{\sqrt{(q-i y)+\tilde{\mu}^{2}}} \frac{G(\tilde{x} a) d \tilde{x}}{\sqrt{(q-i y)^{2}+\tilde{\mu}^{2}-\tilde{x}^{2}}}\right\} .
\end{aligned}
$$

Changing the order of integration over $y$ and $\tilde{x}=x / a$ gives

$$
\begin{aligned}
& \int_{0}^{\infty} d y \cos (\tilde{\varepsilon} y) \int_{0}^{\sqrt{y^{2}+\tilde{\mu}^{2}}} \frac{G(\tilde{x} a)}{\sqrt{y^{2}+\tilde{\mu}^{2}-\tilde{x}^{2}}} d \tilde{x}=\int_{0}^{\tilde{\mu}} d \tilde{x} G(\tilde{x} a) \int_{0}^{\infty} d y \frac{\cos (\tilde{\varepsilon} \tilde{y})}{\sqrt{y^{2}+\tilde{\mu}^{2}-\tilde{x}^{2}}} \\
& +\int_{\tilde{\mu}}^{\infty} d \tilde{x} G(\tilde{x} a) \int_{\sqrt{\tilde{x}^{2}-\tilde{\mu}^{2}}}^{\infty} \frac{\cos (\tilde{\varepsilon} y)}{\sqrt{y^{2}+\tilde{\mu}^{2}-\tilde{x}^{2}}} d y .
\end{aligned}
$$

Since

$$
\begin{aligned}
& \int_{0}^{\infty} \frac{\cos (\tilde{\varepsilon} y) d y}{\sqrt{y^{2}+\tilde{\mu}^{2}-\tilde{x}^{2}}}=K_{0}\left(\tilde{\varepsilon} \sqrt{\tilde{\mu}^{2}-\tilde{x}^{2}}\right) \\
& =-\left(C+\frac{1}{2} \ln \left|\frac{\tilde{\varepsilon}^{2}\left(\tilde{\mu}^{2}-\tilde{x}^{2}\right)}{4}\right|\right)+O\left(\tilde{\varepsilon}^{2} \ln |\tilde{\varepsilon}|\right), \\
& \int_{\sqrt{\tilde{x}^{2}-\tilde{\mu}^{2}}}^{\infty} \frac{\cos (\tilde{\varepsilon} y) d y}{\sqrt{y^{2}+\tilde{\mu}^{2}-\tilde{x}^{2}}}=-\frac{\pi}{2} N_{0}\left(\tilde{\varepsilon} \sqrt{\tilde{x}^{2}-\tilde{\mu}^{2}}\right) \\
& =-\left(C+\frac{1}{2} \ln \left|\frac{\tilde{\varepsilon}^{2}\left(\tilde{x}^{2}-\tilde{\mu}^{2}\right)}{4}\right|\right)+O\left(\tilde{\varepsilon}^{2} \ln |\tilde{\varepsilon}|\right),
\end{aligned}
$$

where $N_{0}(x)$ is Neumann's function, one can obtain

$$
\begin{aligned}
& \int_{0}^{\infty} d y \cos (\tilde{\varepsilon} y) \int_{0}^{\sqrt{y^{2}+\tilde{\mu}^{2}}} \frac{G(\tilde{x} a)}{\sqrt{y^{2}+\tilde{\mu}^{2}-\tilde{x}^{2}}} d \tilde{x} \\
= & -\int_{0}^{\infty} d \tilde{x} G(\tilde{x} a)\left(C+\frac{1}{2} \ln \left|\frac{\tilde{\varepsilon}^{2}\left(\tilde{x}^{2}-\tilde{\mu}^{2}\right)}{4}\right|\right)+O\left(\tilde{\varepsilon}^{2} \ln |\tilde{\varepsilon}|\right) \\
= & -\frac{1}{a} \int_{0}^{\infty} d \eta G(x)\left(C+\frac{1}{2} \ln \left|\frac{\varepsilon^{2} \mu^{2}}{4}\right|+\frac{1}{2} \ln \left|\frac{x^{2}}{\mu^{2}}-1\right|\right)+O\left(\varepsilon^{2} \ln |\varepsilon|\right) .
\end{aligned}
$$


Thus

$$
\begin{aligned}
I_{-}= & -\frac{1}{2} \int_{0}^{\mu} \frac{G(x)}{\sqrt{\mu^{2}-x^{2}}} d x-\frac{1}{a} \int_{0}^{\infty} d x G(x)\left(C+\frac{1}{2} \ln \left|\frac{\varepsilon^{2} \mu^{2}}{4}\right|+\frac{1}{2} \ln \left|\frac{x^{2}}{\mu^{2}}-1\right|\right) \\
& +\frac{1}{a} \int_{\mu}^{\infty} \frac{d u}{e^{2 \pi u / a}-1} \int_{0}^{\sqrt{u^{2}-\mu^{2}}} \frac{[i G(i z)-i G(-i z)]}{\sqrt{u^{2}-\mu^{2}-z^{2}}} d z+O\left(\varepsilon^{2} \ln |\varepsilon|\right) .
\end{aligned}
$$

If we also take in consideration

$$
\begin{gathered}
\int_{0}^{\infty} \frac{x d x}{1+e^{2 \pi x}}=\frac{1}{48}, \quad \int_{0}^{\infty} \frac{x^{3} d x}{1+e^{2 \pi x}}=\frac{7}{1920}, \\
\frac{\partial}{\mu \partial \eta} G(\mu \eta)=\frac{\partial}{\eta \partial \mu} G(\mu \eta),
\end{gathered}
$$

the resulting expressions for $S_{k}^{m}(\varepsilon, \mu)$ can be presented as Eqs. (27)-(130).

\section{Appendix B}

The terms of a fourth WKB order of $\left\langle\varphi^{2}\right\rangle_{\text {ren }}$ are

$$
\begin{aligned}
& 4 \pi^{2}\left\langle\varphi^{2}\right\rangle_{r e n}^{(4)}=\left\{\left[-\frac{3 f^{\prime 2}\left(r^{2}\right)^{\prime 2}}{16 f^{2} r^{2}}+\frac{r^{2} f^{\prime 2} f^{\prime \prime}}{8 f^{3}}-\frac{f^{\prime}\left(r^{2}\right)^{\prime} f^{\prime \prime}}{4 f^{2}}-\frac{r^{2} f^{4}}{32 f^{4}}-\frac{\left(r^{2}\right)^{\prime \prime} f^{\prime}\left(r^{2}\right)^{\prime}}{2 f r^{2}}\right.\right. \\
& -\frac{\left(r^{2}\right)^{\prime 4}}{32 r^{6}}+\frac{\left(r^{2}\right)^{\prime 2} f^{\prime \prime}}{8 f r^{2}}-\frac{r^{2} f^{\prime \prime 2}}{8 f^{2}}-\frac{r^{2} f^{\prime \prime 2}}{8 f^{2}}+\frac{f^{\prime 3}\left(r^{2}\right)^{\prime}}{8 f^{3}}+\frac{f^{\prime}\left(r^{2}\right)^{\prime 3}}{8 f r^{4}}+\frac{\left(r^{2}\right)^{\prime \prime}\left(r^{2}\right)^{\prime 2}}{4 r^{4}} \\
& \left.+\frac{f^{\prime 2}\left(r^{2}\right)^{\prime \prime}}{4 f^{2}}-\frac{\left(r^{2}\right)^{\prime \prime 2}}{2 r^{2}}-\frac{\left(r^{2}\right)^{\prime \prime} f^{\prime \prime}}{2 f}\right] \xi^{2}+\left[\frac{5 r^{2} f^{\prime} f^{\prime \prime \prime}}{48 f^{2}}+\frac{3\left(r^{2}\right)^{\prime \prime} f^{\prime}\left(r^{2}\right)^{\prime}}{16 f r^{2}}+\frac{f^{\prime}\left(r^{2}\right)^{\prime} f^{\prime \prime}}{3 f^{2}}\right. \\
& -\frac{5 f^{\prime}\left(r^{2}\right)^{\prime 3}}{96 f r^{4}}+\frac{\left(r^{2}\right)^{\prime \prime} f^{\prime \prime}}{24 f}+\frac{\left(r^{2}\right)^{\prime}\left(r^{2}\right)^{\prime \prime \prime}}{24 r^{2}}-\frac{r^{2} f^{\prime \prime \prime \prime}}{24 f}-\frac{3\left(r^{2}\right)^{\prime \prime}\left(r^{2}\right)^{\prime 2}}{16 r^{4}}+\frac{5\left(r^{2}\right)^{\prime 4}}{96 r^{6}}-\frac{17 f^{\prime 3}\left(r^{2}\right)^{\prime}}{96 f^{3}} \\
& +\frac{5\left(r^{2}\right)^{\prime \prime 2}}{24 r^{2}}-\frac{\left(r^{2}\right)^{\prime \prime \prime \prime}}{12}+\frac{11 r^{2} f^{\prime 4}}{96 f^{4}}-\frac{f^{\prime \prime \prime}\left(r^{2}\right)^{\prime}}{8 f}-\frac{f^{\prime}\left(r^{2}\right)^{\prime \prime \prime}}{12 f}+\frac{r^{2} f^{\prime \prime 2}}{8 f^{2}}+\frac{f^{\prime 2}\left(r^{2}\right)^{\prime 2}}{16 f^{2} r^{2}} \\
& \left.-\frac{7 r^{2} f^{\prime 2} f^{\prime \prime}}{24 f^{3}}-\frac{\left(r^{2}\right)^{\prime 2} f^{\prime \prime}}{24 f r^{2}}\right] \xi-\frac{49 f^{\prime}\left(r^{2}\right)^{\prime} f^{\prime \prime}}{960 f^{2}}+\frac{\left(r^{2}\right)^{\prime 2} f^{\prime \prime}}{480 f r^{2}}-\frac{\left(r^{2}\right)^{\prime \prime} f^{\prime}\left(r^{2}\right)^{\prime}}{60 f r^{2}}+\frac{f^{\prime}\left(r^{2}\right)^{\prime 3}}{192 f r^{4}} \\
& -\frac{3\left(r^{2}\right)^{\prime \prime 2}}{160 r^{2}}-\frac{11\left(r^{2}\right)^{\prime 4}}{1920 r^{6}}+\frac{13 f^{3}\left(r^{2}\right)^{\prime}}{480 f^{3}}-\frac{3 f^{\prime 2}\left(r^{2}\right)^{\prime 2}}{640 f^{2} r^{2}}+\frac{11 f^{\prime \prime \prime}\left(r^{2}\right)^{\prime}}{480 f}+\frac{f^{\prime}\left(r^{2}\right)^{\prime \prime \prime}}{80 f}-\frac{\left(r^{2}\right)^{\prime}\left(r^{2}\right)^{\prime \prime \prime}}{240 r^{2}} \\
& +\frac{r^{2 \prime \prime \prime \prime}}{80}-\frac{r^{2} f^{\prime} f^{\prime \prime \prime}}{48 f^{2}}+\frac{r^{2} f^{\prime \prime \prime \prime}}{120 f}-\frac{r^{2} f^{\prime \prime 2}}{48 f^{2}}-\frac{7 r^{2} f^{\prime 4}}{320 f^{4}}-\frac{7 f^{\prime 2}\left(r^{2}\right)^{\prime \prime}}{960 f^{2}}+\frac{\left(r^{2}\right)^{\prime \prime} f^{\prime \prime}}{120 f}+\frac{3\left(r^{2}\right)^{\prime \prime}\left(r^{2}\right)^{\prime 2}}{160 r^{4}}
\end{aligned}
$$




$$
\begin{aligned}
& \left.+\frac{13 r^{2} f^{\prime 2} f^{\prime \prime}}{240 f^{3}}\right\} \frac{d^{2} I_{0}(\mu)}{d \mu^{2}}+\left\{\left[-\frac{3 f^{\prime 2}\left(r^{2}\right)^{\prime 2}}{16 f^{2} r^{2}}+\frac{r^{2} f^{\prime 2} f^{\prime \prime}}{8 f^{3}}-\frac{f^{\prime}\left(r^{2}\right)^{\prime} f^{\prime \prime}}{4 f^{2}}-\frac{r^{2} f^{\prime 4}}{32 f^{4}}\right.\right. \\
& -\frac{\left(r^{2}\right)^{\prime \prime} f^{\prime}\left(r^{2}\right)^{\prime}}{2 f r^{2}}-\frac{\left(r^{2}\right)^{\prime 4}}{32 r^{6}}+\frac{\left(r^{2}\right)^{\prime 2} f^{\prime \prime}}{8 f r^{2}}-\frac{r^{2} f^{\prime \prime 2}}{8 f^{2}}+\frac{f^{\prime 3}\left(r^{2}\right)^{\prime}}{8 f^{3}}+\frac{f^{\prime}\left(r^{2}\right)^{\prime 3}}{8 f r^{4}}+\frac{\left(r^{2}\right)^{\prime \prime}\left(r^{2}\right)^{\prime 2}}{4 r^{4}} \\
& \left.+\frac{f^{2}\left(r^{2}\right)^{\prime \prime}}{4 f^{2}}-\frac{\left(r^{2}\right)^{\prime \prime 2}}{2 r^{2}}-\frac{\left(r^{2}\right)^{\prime \prime} f^{\prime \prime}}{2 f}\right] \xi^{2}+\left[\frac{5 r^{2} f^{\prime} f^{\prime \prime \prime}}{48 f^{2}}+\frac{3\left(r^{2}\right)^{\prime \prime} f^{\prime}\left(r^{2}\right)^{\prime}}{16 f r^{2}}+\frac{f^{\prime}\left(r^{2}\right)^{\prime} f^{\prime \prime}}{3 f^{2}}\right. \\
& -\frac{5 f^{\prime}\left(r^{2}\right)^{\prime 3}}{96 f r^{4}}+\frac{\left(r^{2}\right)^{\prime \prime} f^{\prime \prime}}{24 f}+\frac{\left(r^{2}\right)^{\prime}\left(r^{2}\right)^{\prime \prime \prime}}{24 r^{2}}-\frac{r^{2} f^{\prime \prime \prime \prime}}{24 f}-\frac{3\left(r^{2}\right)^{\prime \prime}\left(r^{2}\right)^{\prime 2}}{16 r^{4}}+\frac{5\left(r^{2}\right)^{4}}{96 r^{6}}-\frac{17 f^{\prime 3}\left(r^{2}\right)^{\prime}}{96 f^{3}} \\
& +\frac{5\left(r^{2}\right)^{\prime \prime 2}}{24 r^{2}}-\frac{r^{2^{\prime \prime \prime \prime}}}{12}+\frac{11 r^{2} f^{\prime 4}}{96 f^{4}}-\frac{f^{\prime \prime \prime}\left(r^{2}\right)^{\prime}}{8 f}-\frac{f^{\prime}\left(r^{2}\right)^{\prime \prime \prime}}{12 f}+\frac{r^{2} f^{\prime \prime 2}}{8 f^{2}}+\frac{f^{\prime 2}\left(r^{2}\right)^{\prime 2}}{16 f^{2} r^{2}}-\frac{7 r^{2} f^{\prime 2} f^{\prime \prime}}{24 f^{3}} \\
& \left.-\frac{\left(r^{2}\right)^{\prime 2} f^{\prime \prime}}{24 f r^{2}}\right] \xi-\frac{49 f^{\prime}\left(r^{2}\right)^{\prime} f^{\prime \prime}}{960 f^{2}}+\frac{\left(r^{2}\right)^{\prime 2} f^{\prime \prime}}{480 f r^{2}}-\frac{\left(r^{2}\right)^{\prime \prime} f^{\prime}\left(r^{2}\right)^{\prime}}{60 f r^{2}}+\frac{f^{\prime}\left(r^{2}\right)^{\prime 3}}{192 f r^{4}}-\frac{3\left(r^{2}\right)^{\prime \prime 2}}{160 r^{2}} \\
& -\frac{11\left(r^{2}\right)^{\prime 4}}{1920 r^{6}}+\frac{13 f^{\prime 3}\left(r^{2}\right)^{\prime}}{480 f^{3}}-\frac{3 f^{\prime 2}\left(r^{2}\right)^{\prime 2}}{640 f^{2} r^{2}}+\frac{11 f^{\prime \prime \prime}\left(r^{2}\right)^{\prime}}{480 f}+\frac{f^{\prime}\left(r^{2}\right)^{\prime \prime \prime}}{80 f}-\frac{\left(r^{2}\right)^{\prime}\left(r^{2}\right)^{\prime \prime \prime}}{240 r^{2}} \\
& +\frac{r^{2^{\prime \prime \prime \prime}}}{80}-\frac{r^{2} f^{\prime} f^{\prime \prime \prime}}{48 f^{2}}+\frac{r^{2} f^{\prime \prime \prime \prime}}{120 f}-\frac{r^{2} f^{\prime \prime 2}}{48 f^{2}}-\frac{7 r^{2} f^{\prime 4}}{320 f^{4}}-\frac{7 f^{\prime 2}\left(r^{2}\right)^{\prime \prime}}{960 f^{2}}+\frac{\left(r^{2}\right)^{\prime \prime} f^{\prime \prime}}{120 f} \\
& \left.+\frac{3\left(r^{2}\right)^{\prime \prime}\left(r^{2}\right)^{\prime 2}}{160 r^{4}}+\frac{13 r^{2} f^{\prime 2} f^{\prime \prime}}{240 f^{3}}\right\} \frac{d I_{0}(\mu)}{\mu d \mu}+\left\{\left[\frac{f^{\prime}\left(r^{2}\right)^{\prime} f^{\prime \prime}}{4 f^{2}}-\frac{\left(r^{2}\right)^{\prime 2} f^{\prime \prime}}{8 f r^{2}}+\frac{\left(r^{2}\right)^{\prime 4}}{32 r^{6}}\right.\right. \\
& +\frac{3 f^{\prime 2}\left(r^{2}\right)^{\prime 2}}{16 f^{2} r^{2}}+\frac{\left(r^{2}\right)^{\prime \prime 2}}{2 r^{2}}+\frac{r^{2} f^{\prime \prime 2}}{8 f^{2}}+\frac{r^{2} f^{\prime 4}}{32 f^{4}}-\frac{f^{\prime}\left(r^{2}\right)^{\prime 3}}{8 f r^{4}}-\frac{r^{2} f^{\prime 2} f^{\prime \prime}}{8 f^{3}}-\frac{\left(r^{2}\right)^{\prime \prime}\left(r^{2}\right)^{\prime 2}}{4 r^{4}} \\
& \left.-\frac{f^{\prime 3}\left(r^{2}\right)^{\prime}}{8 f^{3}}+\frac{\left(r^{2}\right)^{\prime \prime} f^{\prime \prime}}{2 f}-\frac{f^{\prime 2}\left(r^{2}\right)^{\prime \prime}}{4 f^{2}}+\frac{\left(r^{2}\right)^{\prime \prime} f^{\prime}\left(r^{2}\right)^{\prime}}{2 f r^{2}}\right] \xi^{2}+\left[-\frac{5\left(r^{2}\right)^{\prime \prime 2}}{24 r^{2}}-\frac{5\left(r^{2}\right)^{\prime 4}}{96 r^{6}}\right. \\
& +\frac{r^{2^{\prime \prime \prime \prime}}}{12}-\frac{r^{2} f^{\prime \prime 2}}{8 f^{2}}+\frac{\left(r^{2}\right)^{\prime 2} f^{\prime \prime}}{24 f r^{2}}+\frac{7 r^{2} f^{\prime 2} f^{\prime \prime}}{24 f^{3}}-\frac{f^{\prime 2}\left(r^{2}\right)^{\prime 2}}{16 f^{2} r^{2}}+\frac{5 f^{\prime}\left(r^{2}\right)^{\prime 3}}{96 f r^{4}}-\frac{5 r^{2} f^{\prime} f^{\prime \prime \prime}}{48 f^{2}} \\
& -\frac{f^{\prime}\left(r^{2}\right)^{\prime} f^{\prime \prime}}{3 f^{2}}+\frac{f^{\prime \prime \prime}\left(r^{2}\right)^{\prime}}{8 f}+\frac{3\left(r^{2}\right)^{\prime \prime}\left(r^{2}\right)^{\prime 2}}{16 r^{4}}+\frac{17 f^{\prime 3}\left(r^{2}\right)^{\prime}}{96 f^{3}}-\frac{11 r^{2} f^{\prime 4}}{96 f^{4}}-\frac{\left(r^{2}\right)^{\prime \prime} f^{\prime \prime}}{24 f} \\
& \left.-\frac{3\left(r^{2}\right)^{\prime \prime} f^{\prime}\left(r^{2}\right)^{\prime}}{16 f r^{2}}+\frac{f^{\prime}\left(r^{2}\right)^{\prime \prime \prime}}{12 f}-\frac{\left(r^{2}\right)^{\prime}\left(r^{2}\right)^{\prime \prime \prime}}{24 r^{2}}+\frac{r^{2} f^{\prime \prime \prime \prime}}{24 f}\right] \xi-\frac{r^{2^{\prime \prime \prime \prime}}}{80}+\frac{3\left(r^{2}\right)^{\prime \prime 2}}{160 r^{2}}
\end{aligned}
$$




$$
\begin{aligned}
& +\frac{11\left(r^{2}\right)^{\prime 4}}{1920 r^{6}}-\frac{13 f^{\prime 3}\left(r^{2}\right)^{\prime}}{480 f^{3}}-\frac{11 f^{\prime \prime \prime}\left(r^{2}\right)^{\prime}}{480 f}-\frac{f^{\prime}\left(r^{2}\right)^{\prime \prime \prime}}{80 f}+\frac{\left(r^{2}\right)^{\prime}\left(r^{2}\right)^{\prime \prime \prime}}{240 r^{2}}-\frac{r^{2} f^{\prime \prime \prime \prime}}{120 f}+\frac{r^{2} f^{\prime \prime 2}}{48 f^{2}} \\
& +\frac{7 r^{2} f^{\prime 4}}{320 f^{4}}+\frac{7 f^{\prime 2}\left(r^{2}\right)^{\prime \prime}}{960 f^{2}}-\frac{\left(r^{2}\right)^{\prime \prime} f^{\prime \prime}}{120 f}-\frac{3\left(r^{2}\right)^{\prime \prime}\left(r^{2}\right)^{\prime 2}}{160 r^{4}}+\frac{49 f^{\prime}\left(r^{2}\right)^{\prime} f^{\prime \prime}}{960 f^{2}}-\frac{\left(r^{2}\right)^{\prime 2} f^{\prime \prime}}{480 f r^{2}} \\
& \left.-\frac{f^{\prime}\left(r^{2}\right)^{\prime 3}}{192 f r^{4}}+\frac{3 f^{\prime 2}\left(r^{2}\right)^{\prime 2}}{640 f^{2} r^{2}}+\frac{r^{2} f^{\prime} f^{\prime \prime \prime}}{48 f^{2}}-\frac{13 r^{2} f^{\prime 2} f^{\prime \prime}}{240 f^{3}}+\frac{\left(r^{2}\right)^{\prime \prime} f^{\prime}\left(r^{2}\right)^{\prime}}{60 f r^{2}}\right\} \frac{1}{\mu^{2}} .
\end{aligned}
$$

[1] M.S. Morris and K.S. Thorne, Wormholes in spacetime and their use for interstellar travel: A tool for teaching general relativity, Am. J. Phys. 56, 395 (1988).

[2] S. Sushkov, Phys. Lett. A, A selfconsistent semiclassical solution with a throat in the theory of gravity, 164, 33 (1992).

[3] D. Hochberg, A. Popov, and S. V. Sushkov, Self-consistent wormhole solutions of semiclassical gravity, Phys. Rev. Lett. 78, 2050 (1997).

[4] A. Popov, Long throat of a wormhole created from vacuum fluctuations, Class. Quantum Grav. 22, 5223 (2005).

[5] A. A. Starobinsky, A new type of isotropic cosmological models without singularity, Phys. Lett. B 91, 99 (1980).

[6] S. G. Mamayev, V. M. Mostepanenko, Isotropic cosmological models determined by vacuum quantum effects, Zh. Eksp. Teor. Fiz. 78, 20 (1980).

[7] L. A. Kofman, V. Sakhni, A. A. Starobinskii, Anisotropic cosmological model created by quantum polarization of vacuum, Sov. Phys. JETP 58, 1090 (1983).

[8] L. A. Kofman, V. Sahni, A new self-consistent solution of the Einstein equations with one-loop quantum-gravitational corrections, Phys. Lett. B 127, 197 (1983).

[9] L. A. Kofman, V. Sahni, Some self-consistent solutions of the Einstein equations with one-loop quantum gravitational corrections: $G_{i k}=8 \pi G\left\langle T_{i k}\right\rangle_{v a c}$, Phys. Lett. A 117, 275 (1986).

[10] P. R. Anderson, W. A. Hiscock, and D. A. Samuel, Stress-energy tensor of quantized scalar fields in static spherically symmetric spacetimes, Phys. Rev. D 51, 4337 (1995).

[11] K. W. Howard and P. Candelas, Quantum stress tensor in Schwarzschild space-time, Phys. Rev. Lett. 53, 403 (1984).

[12] P. Candelas, Vacuum polarization in Schwarzschild spacetime, Phys. Rev. D 21, 2185 (1980). 
[13] M. S. Fawcett, The Energy-Momentum Tensor near a Black Hole, Commun. Math. Phys. 89, 103 (1983).

[14] B. P. Jensen and A. Ottewill, Renormalized electromagnetic stress tensor in Schwarzschild spacetime, Phys. Rev. D 39, 1130 (1989).

[15] B. P. Jensen, J. G. Mc Laughlin, and A. C. Ottewill, Anisotropy of the quantum thermal state in schwarzschild space-time, Phys. Rev. D 45, 3002 (1992).

[16] P. R. Anderson, W. A. Hiscock, and D. J. Loranz, Semiclassical stability of the extreme Reissner-Nordström black hole, Phys. Rev. Lett. 74, 4365 (1995).

[17] E. R. Bezerra de Mello, V. B. Bezerra, and N. R. Khusnutdinov, Vacuum polarization of a massless spinor field in global monopole spacetime, Phys. Rev. D 60, 063506 (1999).

[18] V. P. Frolov and A. I. Zel'nikov, Vacuum polarization by a massive scalar field in Schwarzschild spacetime, Phys. Lett B 115, 372 (1982).

[19] V. P. Frolov and A. I. Zel'nikov, Vacuum polarization of massive fields in Kerr spacetime, Phys. Lett B 123, 197 (1983).

[20] V. P. Frolov and A. I. Zel'nikov, Vacuum polarization of massive fields near rotating black holesPhys. Rev. D 29, 1057 (1984).

[21] R. Herman, A method for calculating the imaginary part of the Hadamard Elementary function $G^{(1)}$ in static, spherically symmetric spacetimes, Phys. Rev. D 58, 084028 (1998).

[22] J. Matyjasek, Stress-energy tensor of neutral massive fields in the Reissner-Nordstrom spacetime, Phys. Rev. D 61, 124019 (2000).

[23] H. Koyama, Y. Nambu, and A. Tomimatsu, Vacuum polarization of massive scalar fields on the black hole horizon, Mod. Phys. Lett. A 15, 815 (2000).

[24] J. Matyjasek, Vacuum polarization of massive scalar fields in the spacetime of the electrically charged nonlinear black hole, Phys. Rev. D 63, 084004 (2001).

[25] D. N. Page, Thermal stress tensors in static Einstein spaces, Phys. Rev. D 25, 1499 (1982).

[26] M. R. Brown and A. C. Ottewill, Effective actions and conformal transformations, Phys. Rev. D 31, 2514 (1985).

[27] M. R. Brown, A. C. Ottewill, and D. N. Page, Conformally invariant quantum field theory in static Einstein space-times, Phys. Rev. D 33, 2840 (1986).

[28] T. Zannias, Renormalized thermal stress tensor for arbitrary static space-times, Phys. Rev. D 30, 1161 (1984). 
[29] V. P. Frolov and A. I. Zel'nikov, Killing approximation for vacuum and thermal stress-energy tensor in static space-times, Phys. Rev. D 35, 3031 (1987).

[30] P. B. Groves, P. R. Anderson, and E. D. Carlson, Method to compute the stress-energy tensor for the massless spin 1/2 field in a general static spherically symmetric spacetime, Phys. Rev. D 66, 124017 (2002).

[31] N. Nakazawa and T. Fukuyama, On the energy-momentum tensor at finite temperature in curved space-time, Nucl. Phys. B 252, 621 (1985).

[32] P. Candelas and K. W. Howard, Vacuum $\left\langle\phi^{2}\right\rangle$ in Schwarzschild spacetime, Phys. Rev. D 29, 1618 (1984).

[33] K. W. Howard, Vacuum $\left\langle T_{\mu}^{\nu}\right\rangle$ in Schwarzschild spacetime, Phys. Rev. D 30, 2532 (1984).

[34] P. R. Anderson, A method to compute $\left\langle\phi^{2}\right\rangle$ in asymptotically flat, static, spherically symmetric spacetimes, Phys. Rev. D 41, 1152 (1990).

[35] A. Popov and O. Zaslavskii, Quantum-corrected ultraextremal horizons and the validity of the WKB approximation in the massless limit, Phys. Rev. D 75, 084018 (2007).

[36] A. A. Popov and S. V. Sushkov, Vacuum polarization of a scalar field in wormhole spacetimes, Phys. Rev. D 63, 044017 (2001).

[37] A. Popov, Local expansion of the bivector of geodesic parallel displacement, Grav. \& Cosm. 13, 119 (2007).

[38] A. A. Popov, Stress-energy of a quantized scalar field in static wormhole spacetimes, Phys. Rev. D 64, 104005 (2001).

[39] M. A. Evgrafov, Analytic Functions (Nauka, Moskow, 1968).

[40] S. V. Sushkov, Analytical approximation of $\left\langle\phi^{2}\right\rangle$ for a massive scalar field in static spherically symmetric spacetimes, Phys. Rev. D 62, 064007 (2000).

[41] N.R. Khusnutdinov, The Abel-Plana formulas, Space, time and fundamental interactions 2, $71(2013)$. 